\title{
MULTI-DOCUMENT SUMMARIZATION Using NEURAL NETWORK
}

\author{
Adesh $^{1}$, Akash $^{2}$ \\ Department of computer engineering, \\ SAOE,Kondhwa(Bk.) \\ Vivek $^{3}$, Abhijeet ${ }^{4}$ \\ Department of computer engineering, \\ SAOE,Kondhwa(Bk.) \\ Prof. N.M.Ranjan \\ Department of computer engineering, \\ SAOE,Kondhwa(Bk.)
}

\begin{abstract}
Multi-document summarization system is employed to summarize many documents into a brief one with generated new sentences. Several of them are supported word-graph and ILP methodology and much of sentences are unnoticed owing to the significant computation load. To reduce computation and generate decipherable and informative summaries, we tend to propose a completely unique multi-document summarization system using Latent Dirichlet Allocation(LDA) model which is widely used topic modeling technique.LDA model is used to classify text documents to a particular topic. Our approach also use the lexrank to score sentences and summaries of documents to generate informative and higher linguistic quality summaries than existing system.
\end{abstract}

Keywords- Summarization, LDA, Sentence level clustering, lexrank

\section{INTRODUCTION}

In order to generate informative and readable summary from multi-document, we propose a Multi-document summarization using neural network, It summarizes several documents into a short one with new generated sentences. We are proposing a summarization system which will create summary of multiple source documents. We give one time period and it will create summarization of all documents related to our topic within a given time period. It ignores irrelevant information but preserves its information content, central ideas and overall meaning of the text. It gives higher linguistic quality summaries which overall saves time of user.

Our approach consists of subsequent four steps. In the first step, documents will be preprocessed using stopword removal and stemming method. These methods are able to remove the unwanted data from documents and help to ignore the irrelevant information. Then using LDA model, the topics per document and words related to topic are extracted. LDA model is an example of topic modeling. This model is able to select representative sentences. After that our approach will generate sentence clusters from given documents. We are able to make the clusters of similar sentences from given documents and eliminate redundancies among documents. Considering the foremost informative sentence ought to be place within the 1st place, therefore we have a tendency to use Lexrank to urge the informative score for every outline of cluster, outputting them in down order per their score because the whole summaries. 


\section{PROBLEM STATEMENT}

Reading and extracting information from large datasets becomes difficult and time consuming. Single document summary captures both the information well but it is not in the case of multi-document summary where the overall comprehensive quality in presenting informative summary often lacks. Most of the existing methods tend to focus on sentence scoring and less consideration is given to the contextual information content in multiple documents.

\section{RELATED WORK}

\subsection{EXISTING SYSTEM}

Automatic multi-document theoretical report system is employed to summarize many documents into a brief one with generated new sentences. Several of them square measure supported word-graph and ILP methodology to compress multiple sentences. The word-graph uses a directed graph to represent the sentences and find the shortest path in the graph as a compressed sentence. In this system, plenty of sentences are ignored because of the heavy computation load.

\subsection{Title and Authors}

Title:-Abstractive Multi-Document Summarization with Semantic Information Extraction. Author:-Wei Li

In this system, concept of BSU is proposed and summary is generated based on semantic network. In this approach when the verbs of BSU's are not meaningful actions, like 'be', the semantic relations between them can not be appropriately computed by the methods described in the papers. The approach is effective in generating informative, coherent and compact summary.

Title:-LCSTS : A Large Scale Chinese Short Text Summarization Dataset.

Author:-Baotian Hu, Quingcai Chen,Fanngze Zhu

In this paper we explore the properties of datasets and sample 10,666 instances for manually checking and scoring the quality of datasets. We take the whole short text as one sequence, this may not be reasonable because most of the short texts contain several sentences.

Title:-Neural Attention Model for Abstractive Sentence Summarization.

Author:-Alexander M Rush, Sumit Chopra, Jason Weston

Neural attention model is structurally simple so it can easily be trained end to end and scales to a large amount of training data. The model shows significant performance gains. Using this model perfect scores for extractive summarization are theoretically, computationally hard to achieve

Title:-Recurrent Neural Network Based Language Model

Author:-Tomas Mikolov, Karafi 'at, L.Burget, J.Cernocky.

This model connects the language modeling more closely to machine learning, data compression and cognitive science researches. The models coming from research tend to be complex and often work well only for systems based on very limited amounts of training data. 


\section{SYSTEM ARCHITECTURE AND METHODOLOGY}

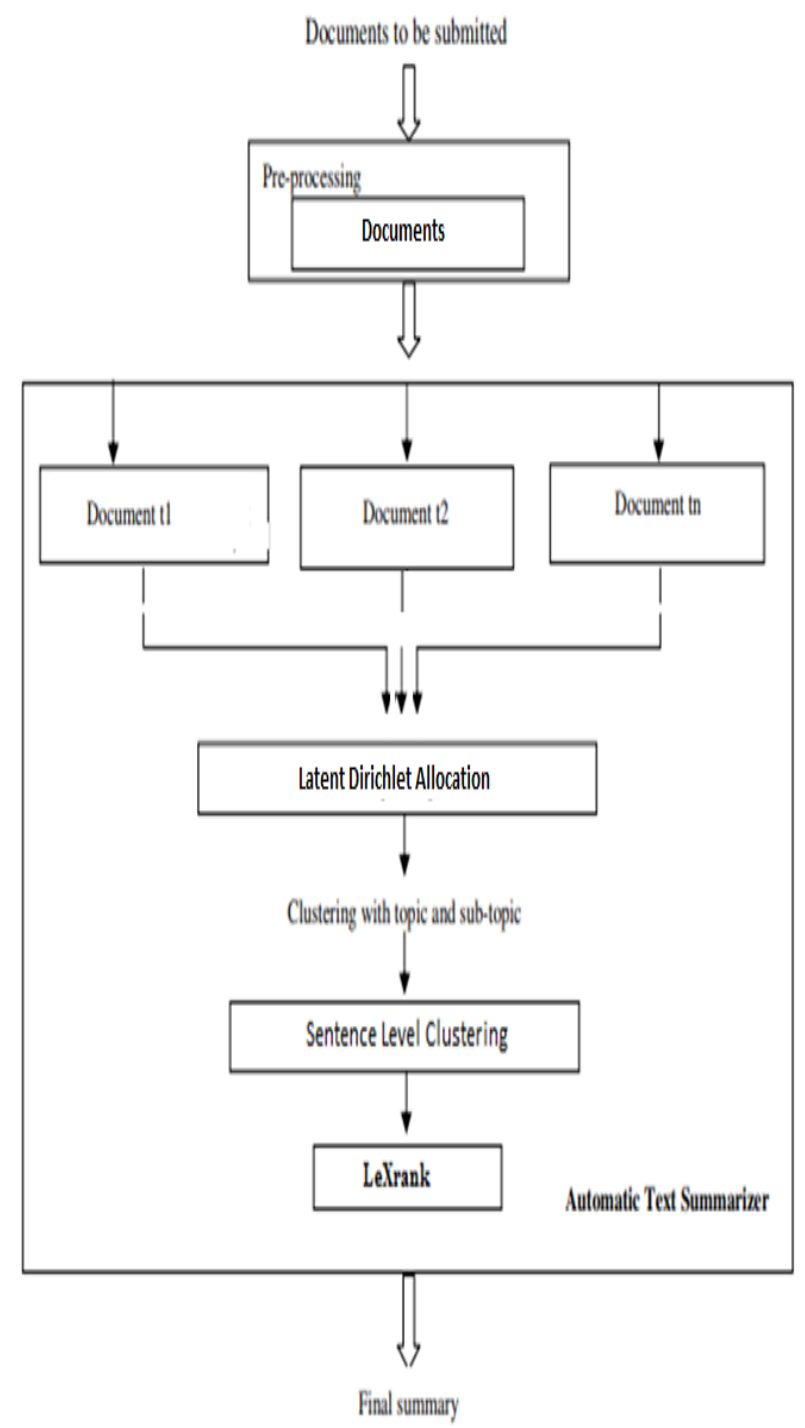

Fig.1 Methodology

\section{AIGORITHM}

\subsection{Latent Dirichlet Allocation}

LDA is widely used topic modeling technique. In the LDA model, each document is viewed as a mixture of topics that are present in the corpus. The model proposes that each word in the document is attributable to one of the document's topics.For example, consider the following set of documents as the corpus:

Document 1: I had a peanut butter sandwich for breakfast.

Document 2: I like to eat almonds, peanuts and walnuts

Document 3: My neighbor got a little dog yesterday.

Document 4: Cats and dogs are mortal enemies.

Document 5: You mustn't feed peanuts to your dog.

LDA model discovers the different topics that the documents represent and how much of each topic is present in a document. For example LDA may produce the following results:

Topic 1: $30 \%$ peanuts, $15 \%$ almonds, $10 \%$ breakfast... ( this topic deals with food) 
DOI: https://dx.doi.org/10.26808/rs.ca.i8v6.07 International Journal of Computer Application (2250-1797)

Issue 8 Volume 6, Nov.- Dec. 2018

Topic 2: $20 \%$ dogs, $10 \%$ cats, $5 \%$ peanuts... ( this topic deals with pets and animals)

$\begin{array}{lllllll}\text { Documents } & \mathbf{1} & \text { and } & \mathbf{2}: & 100 \% & \text { Topic } & 1 \\ \text { Documents } & \mathbf{3} & \text { and } & \mathbf{4} & 100 \% & \text { Topic } & 2\end{array}$

Document 5: 70\% Topic 1, 30\% Topic 2

\subsection{Sentence Level Clustering}

Clustering is a task of grouping a set of objects in a such way that objects in a same groupe are more similar to each other than to those in other groups

Step 1: Enter the user question.(e.g., what's java.)

Step 2: Apply Steaming and stopping (e.g., take away stop words.Here during this example, 'what' and 'is' words are going to be removed)

Step 3: Remaining words are going to be search within the another document get in info.

Step 4: result are going to be displayed as sentences wherever the keyword is gift.

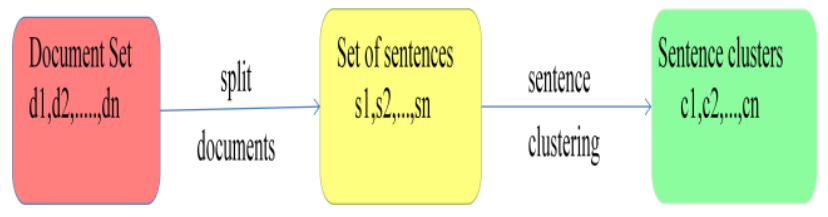

\subsection{LEXRANK}

1. In the end, we use LexRank to score all summaries generate.

2. We use lexRank algorithm to assign each sentence cluster's summary a score which represents how many information the summary contains.

3. LexRank calculate all sentences iteratively.

4. Then we output all sentence clusters summaries in descending order of their lexRank score.

\section{CONCLUSION}

The proposed system provides a general survey on multi document summarization approaches which are tailored in a way that researchers whom are new to the area of text summarization can grasp the idea of various multi document summarization approaches. Our summarization system will create informative, readable and high linguistic quality summaries than existing systems. It outperforms all baseline systems and reach the state of art systems. Our proposing system tends to decrease computational load and eliminate sentence redundancies to make it readable and save user's time.

\section{ACKNOWLEDGMENTS}

We want to acknowledge Principal, Head of department and guide of their project for all the support and help rendered. To express profound feeling of appreciation to their regarded guardians for giving the motivation required to the finishing of paper.

\section{REFERENCES}

[1] G. Carenini and J. C. K. Cheung, "Extractive vs. nlg-based abstractive summarization of evaluative text: The effect of corpus controversiality," in Proceedings of the Fifth International Natural Language Generation Conference. Association for Computational Linguistics, 2008, pp. 33- 41.

[2] T. Mikolov, M. Karafi'at, L. Burget, J. Cernock'y, and S. Khudanpur, "Recurrent neural network based language model." in Interspeech, vol. 2, 2010, p. 3. 
DOI: https://dx.doi.org/10.26808/rs.ca.i8v6.07 International Journal of Computer Application (2250-1797)

Issue 8 Volume 6, Nov.- Dec. 2018

[3] K. Filippova, "Multi-sentence compression: finding shortest paths in word graphs," in Proceedings of the 23rd International Conference on Computational Linguistics. Association for Computational Linguistics, 2010, pp. 322-330.

[4] C.-Y. Lin, "Rouge: A package for automatic evaluation of summaries," in Text summarization branches out: Proceedings of the ACL-04 workshop. Barcelona, Spain, 2004.

[5] S. Banerjee, P. Mitra, and K. Sugiyama, "Multi-document abstractive summarization using ilp based multi-sentence compression," in Proceedings of the 24th International Conference on Artificial Intelligence. AAAI Press, 2015, pp. 1208-1214.

[6] W. Li, "Abstractive multi-document summarization with semantic information extraction," in Proceedings of the 2015 Conference on Empirical Methods in Natural Language Processing, 2015, pp. 1908-1913.

[7] B. Hu, Q. Chen, and F. Zhu, "Lcsts: A large scale chinese short text summarization dataset," arXiv preprint arXiv:1506.05865, 2015.

[8] A. M. Rush, S. Chopra, and J. Weston, "A neural attention model for abstractive sentence summarization," arXiv preprint arXiv:1509.00685, 2015.

[9] D. Bahdanau, K. Cho, and Y. Bengio, "Neural machine translation by jointly learning to align and translate," arXiv preprint arXiv:1409.0473, 2014.

[10] J. Gu, Z. Lu, H. Li, and V. O. Li, "Incorporating copying mechanism in sequence-tosequence learning," arXiv preprint arXiv:1603.06393, 2016.

[11] S. Bird, "Nltk: the natural language toolkit," in Proceedings of the COLING/ACL on Interactive presentation sessions. Association for Computational Linguistics, 2006, pp. 6972.

[12] K. Toutanova, D. Klein, C. Manning et al., "Stanford core nlp," The Stanford Natural Language Processing Group. Available: http://nlp. stanford. edu/software/corenlp. shtml. Accessed, 2013.

[13] G. Erkan and D. R. Radev, "Lexrank: Graph-based lexical centrality as salience in text summarization," Journal of Artificial Intelligence Research, vol. 22, pp. 457-479, 2004.

[14] T. Mikolov, I. Sutskever, K. Chen, G. S. Corrado, and J. Dean, "Distributed representations of words and phrases and their compositionality," in Advances in neural information processing systems, 2013, pp. 3111-3119.

[15] T. Mikolov, K. Chen, G. Corrado, and J. Dean, "Efficient estimation of word representations in vector space," arXiv preprint arXiv:1301.3781, 2013.

[16] A. Rodriguez and A. Laio, "Clustering by fast search and find of density peaks," Science, vol. 344, no. 6191, pp. 1492-1496, 2014.

[17] E. Parzen, "On estimation of a probability density function and mode," The annals of mathematical statistics, vol. 33, no. 3, pp. 1065-1076,1962.

[18] Y. Bengio, R. Ducharme, P. Vincent, and C. Jauvin, "A neural probabilistic language model," Journal of Machine Learning Research, vol. 3, pp. 1137-1155, Feb 2003. 